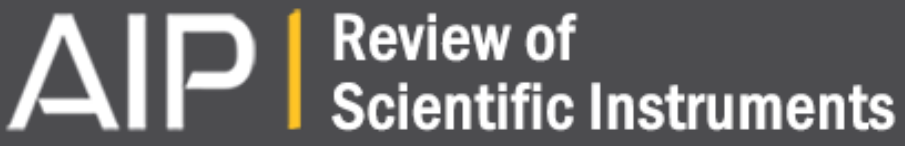

A flexible apparatus for attosecond photoelectron spectroscopy of solids and surfaces

E. Magerl, S. Neppl, A. L. Cavalieri, E. M. Bothschafter, M. Stanislawski, Th. Uphues, M. Hofstetter, U.

Kleineberg, J. V. Barth, D. Menzel, F. Krausz, R. Ernstorfer, R. Kienberger, and P. Feulner

Citation: Review of Scientific Instruments 82, 063104 (2011); doi: 10.1063/1.3596564

View online: http://dx.doi.org/10.1063/1.3596564

View Table of Contents: http://scitation.aip.org/content/aip/journal/rsi/82/6?ver=pdfcov

Published by the AIP Publishing

\section{Articles you may be interested in}

Spin-resolved photoelectron spectroscopy using femtosecond extreme ultraviolet light pulses from high-order harmonic generation

Rev. Sci. Instrum. 87, 043903 (2016); 10.1063/1.4946782

Femtosecond time-resolved photoelectron spectroscopy with a vacuum-ultraviolet photon source based on laser high-order harmonic generation

Rev. Sci. Instrum. 82, 063114 (2011); 10.1063/1.3600901

Retrieval of satellite pulses of single isolated attosecond pulses

Appl. Phys. Lett. 94, 161112 (2009); 10.1063/1.3125247

Control of single attosecond pulse generation from the reflection of a synthesized relativistic laser pulse on a solid surface

Phys. Plasmas 15, 103107 (2008); 10.1063/1.2997342

Tunable pulsed vacuum ultraviolet light source for surface science and materials spectroscopy based on high order harmonic generation

Rev. Sci. Instrum. 72, 1977 (2001); 10.1063/1.1351835

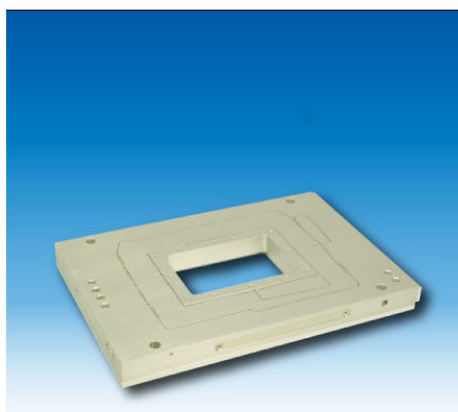

Nanopositioning Systems

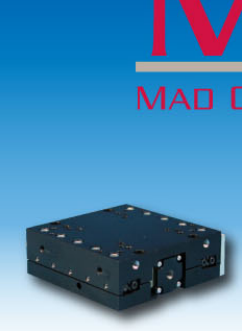

Micropositioning

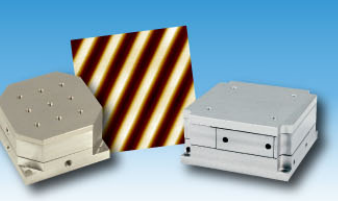

AFM \& SPM

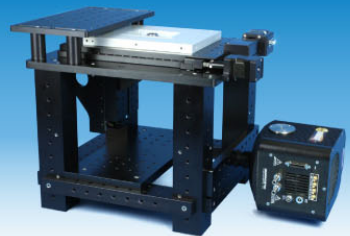

Single molecule imaging 


\title{
A flexible apparatus for attosecond photoelectron spectroscopy of solids and surfaces
}

\author{
E. Magerl, ${ }^{1}$ S. Neppl, ${ }^{2}$ A. L. Cavalieri, ${ }^{1,3}$ E. M. Bothschafter ${ }^{1,4}$ M. Stanislawski, ${ }^{1}$ \\ Th. Uphues, ${ }^{1}$ M. Hofstetter, ${ }^{1,5}$ U. Kleineberg, ${ }^{1,5}$ J. V. Barth, ${ }^{2}$ D. Menzel, ${ }^{2}$ F. Krausz, ${ }^{1,5}$ \\ R. Ernstorfer, ${ }^{1,4}$ R. Kienberger, ${ }^{1,4}$ and P. Feulner ${ }^{2}$ \\ ${ }^{1}$ Max-Planck-Institut für Quantenoptik, Hans-Kopfermann-Straße 1, 85748 Garching, Germany \\ ${ }^{2}$ Physik Department E20, Technische Universität München, James-Franck-Straße, 85748 Garching, Germany \\ ${ }^{3}$ Max-Planck Research Department for Structural Dynamics, Universität Hamburg, Notkestrasse 85, \\ 22607 Hamburg, Germany \\ ${ }^{4}$ Physik Department E11, Technische Universität München, James-Franck-Straße, 85748 Garching, Germany \\ ${ }^{5}$ Ludwig-Maximilians-Universität München, Fakultät für Physik, Am Coulombwall 1, 85748 Garching, \\ Germany
}

(Received 13 January 2011; accepted 12 May 2011; published online 15 June 2011)

\begin{abstract}
We describe an apparatus for attosecond photoelectron spectroscopy of solids and surfaces, which combines the generation of isolated attosecond extreme-ultraviolet (XUV) laser pulses by high harmonic generation in gases with time-resolved photoelectron detection and surface science techniques in an ultrahigh vacuum environment. This versatile setup provides isolated attosecond pulses with photon energies of up to $140 \mathrm{eV}$ and few-cycle near infrared pulses for studying ultrafast electron dynamics in a large variety of surfaces and interfaces. The samples can be prepared and characterized on an atomic scale in a dedicated flexible surface science end station. The extensive possibilities offered by this apparatus are demonstrated by applying attosecond XUV pulses with a central photon energy of $\sim 125 \mathrm{eV}$ in an attosecond streaking experiment of a xenon multilayer grown on a $\operatorname{Re}(0001)$ substrate. () 2011 American Institute of Physics. [doi:10.1063/1.3596564]
\end{abstract}

\section{INTRODUCTION}

Over the last decades, time-resolved photoelectron spectroscopy utilizing laser pulses reaching from the infrared to the ultraviolet (UV) spectral range has evolved into a tool to routinely study chemical and electronic processes at surfaces on the femtosecond timescale. ${ }^{1-3}$ However, many fundamental phenomena including dynamical electronic screening, 4,5 electron transfer from chemisorbates, ${ }^{6,7}$ and atomic-scale motion of electrons in or near surfaces ${ }^{8}$ proceed on an attosecond timescale, which cannot be resolved even if interferometric two-photon photoelectron spectroscopy with wellcharacterized femtosecond laser pulses are employed. ${ }^{9-11}$

Alternatively, the attosecond transient recorder (ATR) is a newly established technique that provides direct timeresolved access to these processes. The ATR uses carrierenvelope phase (CEP)-stabilized few-cycle laser pulses in the near infrared (NIR) to reproducibly modulate or "streak" the kinetic energy of photoelectrons generated by a synchronized isolated sub-fs XUV pulse. ${ }^{12,13}$ This technique has been used extensively to study attosecond electron dynamics in atoms in the gas phase, ${ }^{13-16}$ and recently the method has been generalized to include condensed matter systems. ${ }^{8}$

We note that photon energies in the extreme-ultraviolet (XUV) range allow excitation of innershell levels, whereas time-resolved photoelectron spectroscopy in the UV range is restricted to investigations of valence states. Valence orbitals depend on chemistry; in most cases, they are delocalized over several or even many atoms. Innershell levels, however, are elemental fingerprints; their binding energies are only moderately affected by the environment; apart from highly symmetric molecules, delocalization can be neglected. In contrast to time-resolved photoelectron spectroscopy in the UV range, the XUV approach allows a site-selective, atom-specific look. Several methods are under development trying to combine high time resolution with high photon energies. ${ }^{17-19}$ Currently, our ATR detection scheme applied to solid surfaces enables photon energies up to $140 \mathrm{eV}$ and time-resolution in the attosecond regime.

Here, we describe a flexible apparatus that enables systematic studies of attosecond phenomena in well-ordered solid state model systems by merging XUV pulse production through high-order harmonic generation (HHG) with standard surface science preparation and detection schemes in ultrahigh vacuum (UHV). In comparison to an apparatus for gas phase streaking experiments, ${ }^{15,20}$ an apparatus designed for condensed matter experiments must satisfy additional requirements. One challenge is the combination of HHG, in a relatively high-density noble gas target, ${ }^{21}$ with surface sensitive photoelectron spectroscopy. ${ }^{22}$ In order to exclude the influence of surface contamination during typical data acquisition periods, including measurements at cryogenic temperatures, a background pressure of $\leq 10^{-10} \mathrm{mbar}$ in the measurement chamber area is needed. Since the pressure inside the HHG target exceeds 100 mbar, and because even noble gases, with the exception of helium, would adsorb on the sample at cryogenic conditions, a gradient of many orders of magnitude in pressure must be achieved by differential pumping. Further design goals include: tunable XUV photon energies, independent control of the angle of incidence of the laser pulses and the detection angle of the photoelectrons, compatibility with state-of-the-art surface preparation methods in UHV and the possibility for gas phase measurement for calibration and reference purposes. 

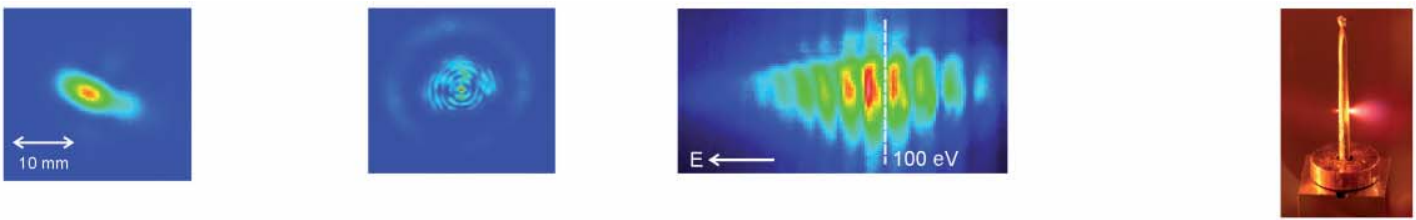

\section{$\mathrm{HH}$ double focus pellicle XUV differential skimmer HHG focussing observation mirror observation slider spectrometer pumping | target mirror}

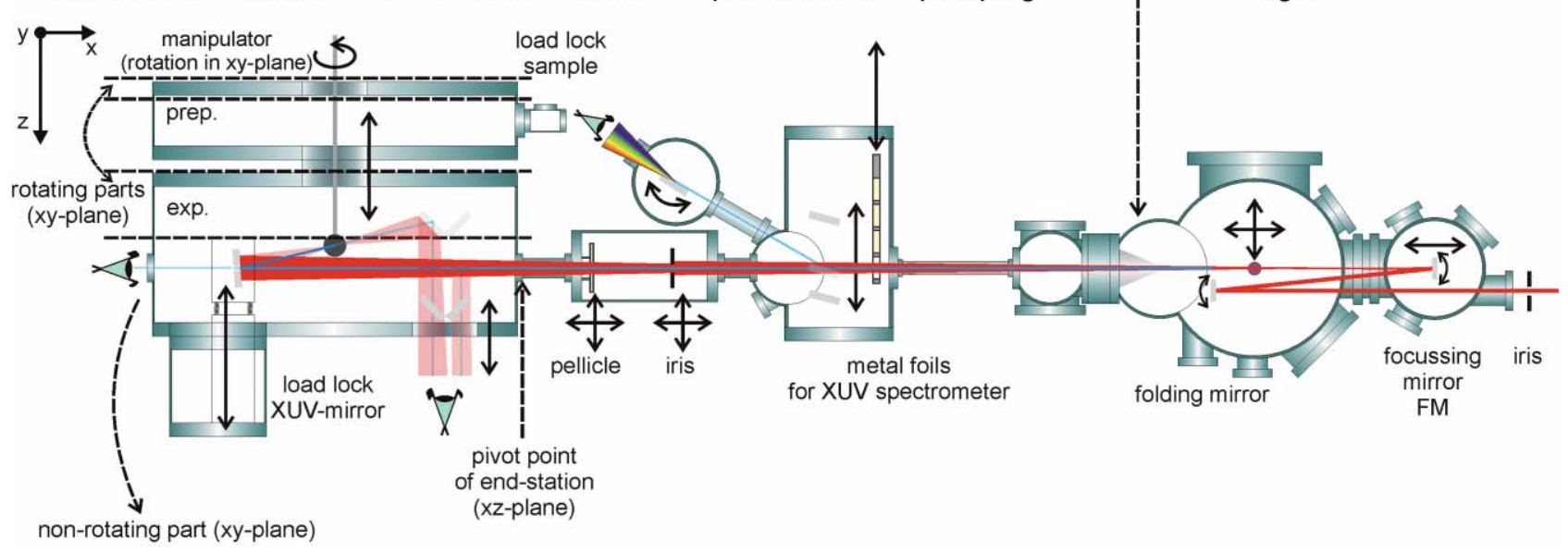

FIG. 1. (Color online) Schematic top-view of the AS-3 beamline and end-station. Details of the various degrees of freedom of sample, preparation tools, detectors, and the end-station are given in Fig. 3. The back plate of the preparation chamber is rotatable so that the manipulator with the sample can be moved to different ports for target preparation and characterization before the sample is inserted into the measurement chamber.

Different approaches for time- and angle-resolved photoelectron spectroscopy, employing femtosecond high harmonic radiation, have been described previously. ${ }^{23-25}$ In contrast, the apparatus described here utilizes attosecond XUV pulses and corresponding attosecond time resolution, increased XUV photon energy range, and enhanced flexibility regarding angle of incidence and detection angle.

In order to meet these requirements, a new attosecond beamline and measurement end station at the Max Planck Institute of Quantum Optics, referred to as AS-3, has been built. To the best of our knowledge, this is currently the only operational beamline providing attosecond time resolution for solid state experiments in UHV. A schematic overview of this apparatus is shown in Fig. 1 and a more detailed description of the beamline and the end station is given below.

\section{BEAMLINE}

A $3 \mathrm{kHz}$ titanium-sapphire laser system in conjunction with hollow-core fiber spectral broadening provides CEPstable, $400 \mu \mathrm{J}$, octave-spanning pulses centered at $750 \mathrm{~nm}$. These spectrally broadened pulses are subsequently compressed to less than $4 \mathrm{fs}$ in a negative dispersion chirped mirror pulse compressor and are ideal for use in HHG. ${ }^{10}$ Following compression, the laser propagates in an evacuated beam tube ( $\mathrm{p}<10^{-3}$ mbar) and enters the AS-3 beamline through an adjustable iris aperture (Fig. 1). The laser pulses are focused onto the HHG target by a spherical mirror (FM) in a z-folded geometry. The focal length of the optic can be varied from 300 to $800 \mathrm{~mm}$, resulting in peak intensities from $\sim 2 \times 10^{14} \mathrm{~W} / \mathrm{cm}^{2}$ to $\sim 2 \times 10^{15} \mathrm{~W} / \mathrm{cm}^{2}$, respectively. Highharmonic radiation is generated in a small volume of noble gas, typically Ne, with a pressure of $\sim 100$ mbar. The gas is confined by a nickel tube with an inner diameter of $\sim 2 \mathrm{~mm}$. The tube is sealed on one end and supplied with a constant gas pressure from the other, as described before. ${ }^{26}$ The target is mounted on two translation stages for precise alignment of the HHG target along the longitudinal and one transverse axis of the laser beam. Two apertures in the nickel tube along the beam axis are created by laser ablation. The inner-diameter of the nickel tube is adjusted for optimal phase matching conditions for HHG and maximum XUV flux. ${ }^{27,28}$ The laser intensity and the gas pressure of the HHG gas target are also adjusted for optimizing the generation of cut-off harmonics in the range from 90 to $140 \mathrm{eV}$ (see Fig. 2) - in combination

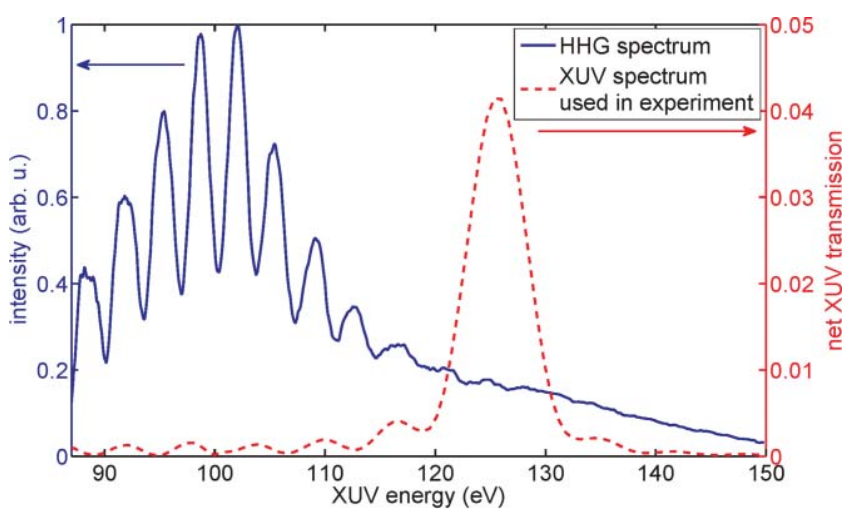

FIG. 2. (Color online) Measured high harmonic spectrum (corrected for reflectivity of the grating and metal filter transmission of $950 \mathrm{~nm}$ of $\mathrm{Zr}$ ) (solid curve). Calculated net XUV transmission used in the reported xenon experiment (based on XUV mirror reflectivity and transmission of a $200 \mathrm{~nm} \mathrm{Zr}$ metal filter) (dotted curve). 
with the CEP-stabilization of the NIR laser a prerequisite for the generation of isolated attosecond pulses in this spectral region. ${ }^{29-31}$ Resultant XUV radiation can be monitored with a home-built spectrometer for the energy region between $80 \mathrm{eV}$ and $140 \mathrm{eV}$. Two grazing incidence gold-palladium mirrors are mounted on a translation stage with direction of motion perpendicular to the beam path. One mirror reflects the direct beam for alignment purposes; the other reflects the XUV beam onto a grating, which disperses the radiation horizontally. A gold-coated flat-field grating (Hitachi No. 001-0437 (Refs. 32 and 33)) suitable for photon energies between 60 and $250 \mathrm{eV}$ is used for dispersion. The detector unit consists of a double microchannel plate assembly in chevron geometry with cesium iodide coating for an enhanced XUV sensitivity and a phosphor screen on the backside for optical detection (Beam Imaging Solutions). Two stages of spectral and spatial filters can be moved in front of the spectrometer. The first stage consists of a $450 \mathrm{~nm}$ thick zirconium foil used for suppression of the fundamental driving laser. A second stage consists of a sliding filter plate, offering four insets for additional filters: free-standing foils of $150 \mathrm{~nm}$ thick silicon, $200 \mathrm{~nm}$ thick aluminum, $200 \mathrm{~nm}$ thick palladium or zirconium, and a $500 \mu \mathrm{m}$ wide slit to improve the resolution of the spectrometer. Figure 2 shows the spectrum of the broadband XUV radiation resulting from $\mathrm{HHG}$ in Ne using a CEP-stabilized, sub-4 fs NIR driving laser pulse. The peak intensity in the HHG target gas was $\sim 5.7 \times 10^{14} \mathrm{~W} / \mathrm{cm}^{2}$.

The gas load emerging from the HHG target divides the beamline in a high vacuum part ( $\mathrm{HV}, 10^{-2}-10^{-9}$ mbar) near the gas target and an ultrahigh vacuum section $\left(<10^{-9} \mathrm{mbar}\right)$ near the end-station. Most of the HHG target gas load is pumped by a 6001 turbo pump directly attached to the HHG chamber. Behind the HHG chamber, a differential pumping stage is realized by a skimmer with an opening of $1.5 \mathrm{~mm}$ and a 2001 turbo pump limiting the downstream gas flow. From here a narrow beam pipe in combination with a 3001 turbo pump is sufficient to keep the pressure below $5 \times 10^{-9}$ mbar. The final transition to a pressure of $<10^{-10}$ mbar is accomplished by an additional differential pumping stage that is pumped by a 4001 turbo pump. This last differential stage does not contain sensitive components and can be baked. In addition, a two component pellicle/Zr foil filter, which is used to separate the XUV and NIR pulses as described below, can be placed in front of the last differential pumping stage and acts as an additional barrier to residual gas emerging from the beamline.

Attosecond streaking experiments require isolated attosecond pulses, filtered from broadband high harmonic radiation, ${ }^{12}$ to be additionally isolated from the driving fundamental NIR pulses. Once separated, the relative timing between the isolated XUV pulse and the NIR pulse must also be precisely adjusted. A two-component pellicle/Zr filter, in combination with a precision-controlled double-mirror assembly, is used for this purpose. ${ }^{26}$ The two-component filter consists of a nitrocellulose pellicle (thickness: 2,5 , or $15 \mu \mathrm{m}$ ) whose inner part has been replaced by a $200 \mathrm{~nm}$ thick zirconium or palladium metal filter with a diameter of $5 \mathrm{~mm}$. When the co-propagating XUV and NIR beams are incident on this filter, the central metal component suppresses the NIR by ap- proximately seven orders of magnitude, effectively blocking it $^{34,35}$ while transmitting a significant fraction of the XUV radiation (the transmission is $52 \%$ at $100 \mathrm{eV}$ for $200 \mathrm{~nm}$ of zirconium and $37 \%$ at $135 \mathrm{eV}$ for $200 \mathrm{~nm}$ of palladium ${ }^{36}$ ). On the other hand, the outer nitrocellulose component allows for nearly full transmission of the NIR $(\sim 92 \%)$, while completely blocking the XUV radiation. Furthermore, due to the fact that the XUV radiation has a much smaller divergence than the NIR radiation, almost all of the XUV radiation, at the relevant photon energies, is incident on the metal, transparent portion of the filter, allowing for a very efficient method for isolation.

The two-component filter is mounted in a sealed translation sleeve for vacuum separation, and can be moved in the plane perpendicular to the beam path for proper alignment and therefore, maximum transmission of the XUV beam. Directly in front of the pellicle, a motorized iris on a three-axis manipulator is used to adjust the intensity of the co-propagating NIR pulse incident on the sample. Behind the pellicle, the annular NIR pulse and the central XUV beam enter the end station (see Sec. III) where they are reflected by a coaxial assembly of two focusing spherical mirrors consisting of a central XUV-reflecting part, typically $5 \mathrm{~mm}$ diameter, and an NIRreflecting outer mirror. ${ }^{26}$ The multilayer surface structure of the inner mirror is designed to reflect XUV radiation with a given bandwidth and central photon energy, which defines the pulse duration of the isolated attosecond pulse. ${ }^{37,38}$ In conjunction with the metal foil in the two-component filter, it reflects a part of the cut-off region of the spectrum with a flat phase, to avoid temporal dispersion, which would broaden the attosecond XUV pulse. ${ }^{38}$ The outer mirror is typically coated with $13 \mathrm{~nm}$ of boron carbide acting as a partial reflector for the NIR pulses with a reflectivity of $\sim 20 \%$. The pointing of the outer mirror is motorized, enabling spatial overlap of the focused beams. Temporal delay between the XUV and the NIR pulses is adjusted by translating the inner mirror along the incident beam with a piezo-driven translation stage, which has a minimum step size of $0.2 \mathrm{~nm}$ corresponding to $\sim 1.3$ attoseconds of delay.

The entire double mirror assembly is mounted on a stack of 4 piezo-driven stages (Nanomotion), which allow positioning of the mirrors relative to the laser beams as well as aligning the reflected beams onto the solid sample. The complete mirror stack can be retracted from the main experimental chamber into a load-lock in order to make exchanging the mirrors possible without breaking vacuum in the main chamber.

\section{END STATION}

The end-station consists of two cylindrical stainless steel vessels separated by a gate valve. One of the chambers is used for sample preparation and characterization and the other for photoelectron spectroscopy (measurement chamber). The longitudinal axes of the chambers are parallel. The measurement chamber is designed in an in-axis geometry, i.e., the sample, the laser focus, and the foci of all detectors in working position coincide with the chamber axis (see below). Offaxis mounting is used for the preparation chamber such that the foci of the preparation/diagnostic instruments lie on a 
circle of $250 \mathrm{~mm}$ radius about the chamber axis for maximum solid-angle range of each station. The sample is brought to the individual prep-stations by moving the manipulator along this circle by means of a large differentially pumped rotary feedthrough. The preparation chamber accommodates standard equipment for surface preparation and controlled layer growth: an ion sputter gun, a low-energy electron diffraction system, LEED (SPECS), a Knudsen effusion cell, a modified Bayard-Alpert pressure gauge for temperature programmed desorption (TPD) studies, and a gas dosing system composed of a multi-capillary array doser attached to a gas manifold via a flow-controller.

The samples are mounted on a holder clamped onto the head of a home-built XYZ-manipulator. A second two-stage differentially pumped feedthrough at the manipulator head enables $360^{\circ}$ rotation around the $\mathrm{Z}$ axis. The sample temperature is controlled with a thermocouple. The reference junction of this thermocouple is attached to the sample holder, the temperature of which is measured independently by a second $\mathrm{K}$ type thermocouple. This design does not require feedthroughs from thermocouple materials at the vacuum interface and allows application of any thermocouple combination as dictated by the requirements of the experiment. An available temperature range of $15-2500 \mathrm{~K}$ is achieved by thermal contact to a liquid $\mathrm{He}$ flow-cryostat integrated in the manipulator, and by radiative or electron-beam heating of the sample. Arbitrary temperature profiles can be generated by software with a proportional integral derivative (PID) -controlled feedback loop. Fast sample exchange without degradation of the UHV conditions is provided by a load lock system.

The measurement chamber is composed of a detector ring that is rotatable around its longitudinal $\mathrm{Z}$ axis (enabled by two differentially pumped rotary feedthroughs) and a fixed section (see Fig. 1). The rotating part is equipped with an electron time-of-flight spectrometer (Stefan Kaesdorf, Geraete fuer Forschung und Industrie), a hemispherical electron energy analyzer HEA (SPECS), and a Mg/Al-twin anode x-ray source (PSP Vacuum Technology) for standard $\mathrm{CW}$ photoelectron spectroscopy for sample characterization. These components are all equipped with translation and tilt mechanisms enabling optimal alignment with the sample surface (see Fig. 3).

This flexible configuration, combined with the sample rotation allows independent choice of both XUV/NIR angle of incidence and of the electron detection angle (see Fig. 3). Considering the linear polarization of both the NIR and the XUV pulses, this arrangement can be used to distinguish polarization from angular effects in the photoemission process.

The NIR and XUV beams enter the end station through the fixed part of the measurement chamber and are reflected by the double mirror assembly onto the sample. For optimization and alignment purposes, the fixed part also houses an $\mathrm{MCP} /$ fluorescent screen system (see above) to observe the XUV beam. In normal operation, this detector is obscured by the double mirror and the chamber wall; therefore, the entire end-station can be rotated horizontally around the entry port using air pads in order to send the beam to this detector. Furthermore, to enable gas phase measurements, a gas nozzle supported by an XYZ-manipulator mounted on the fixed

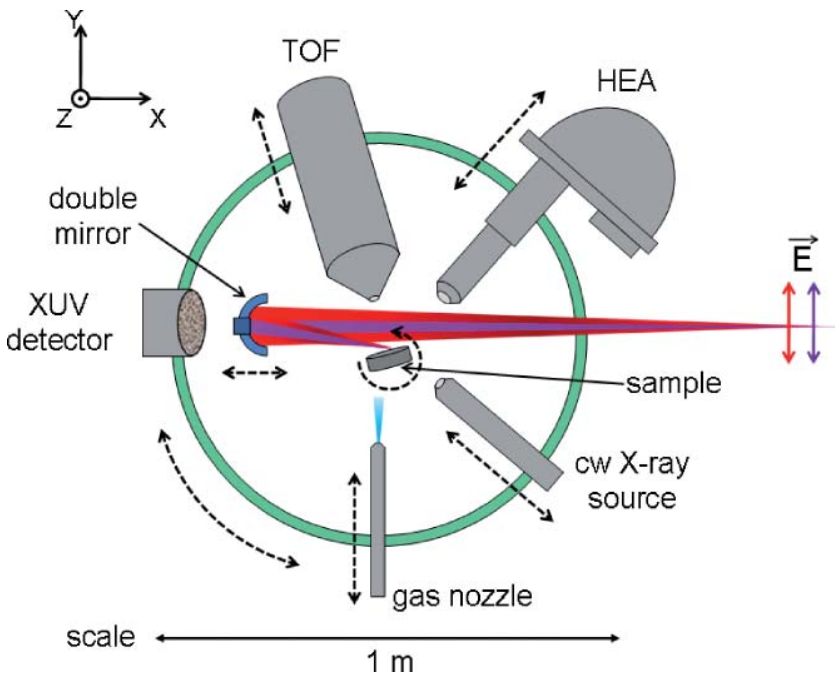

FIG. 3. (Color online) Cross-sectional view of the rotary part of the measurement chamber. The various degrees of freedom for positioning are indicated by dashed arrows.

part can be brought to the center of the chamber to replace the solid sample.

Both chambers and the rotary feedthroughs are individually pumped by a system of turbomolecular and scroll pumps. In addition, the chambers are also pumped by liquid nitrogen cooled titanium sublimation pumps. After baking the UHV system and with the use of $1-\mathrm{N}_{2}$-cooled titanium sublimation pumps, a base pressure below $10^{-10}$ mbar can be routinely achieved and maintained during the measurements.

\section{STREAKING OF CONDENSED XENON}

A xenon multilayer serves as a benchmark system to demonstrate the performance of the new apparatus. Xenon is condensed onto a clean $\operatorname{Re}(0001)$ surface cooled to $20 \mathrm{~K}$ with a final coverage of 200 layers. The xenon coverage has been calibrated by comparing the areas of multilayer and monolayer desorption maxima in TPD. ${ }^{39}$ The atomically clean and well ordered $\operatorname{Re}(0001)$ surface was produced according to established procedures consisting of $\mathrm{Ne}^{+}$sputtering followed by several oxidation and annealing cycles and final flashes to $2400 \mathrm{~K}^{40}$

A MoSi-multilayer mirror acting as a $6.2 \mathrm{eV}$ broad bandpass filter centered at $125 \mathrm{eV}$ filters the XUV continuum produced by HHG and focuses it onto the sample. Photoelectron spectra from 200 atomic layers of xenon taken at different relative delays are shown as a red and a blue curve in Fig. 4(a). Photoemission from the Re substrate is suppressed by the Xe adsorbate and two emission lines at kinetic energies of $\sim 115 \mathrm{eV}$ and $\sim 60 \mathrm{eV}$ with respect to the vacuum level, originating from the $\mathrm{Xe}$ valence band, which is derived from Xe $5 p$ electrons, and $\mathrm{Xe} 4 d_{5 / 2,3 / 2}$ core levels, appear. Emission from the Xe $5 s$ state cannot be resolved due to its low cross section. The weak features in the region between the two visible Xe-states are due to satellite reflections of the multilayer mirror (cf. Fig. 2). The peak widths of the Xe states reflect the broad energy range of the exciting attosecond XUV pulse and the energetic structure of the Xe 

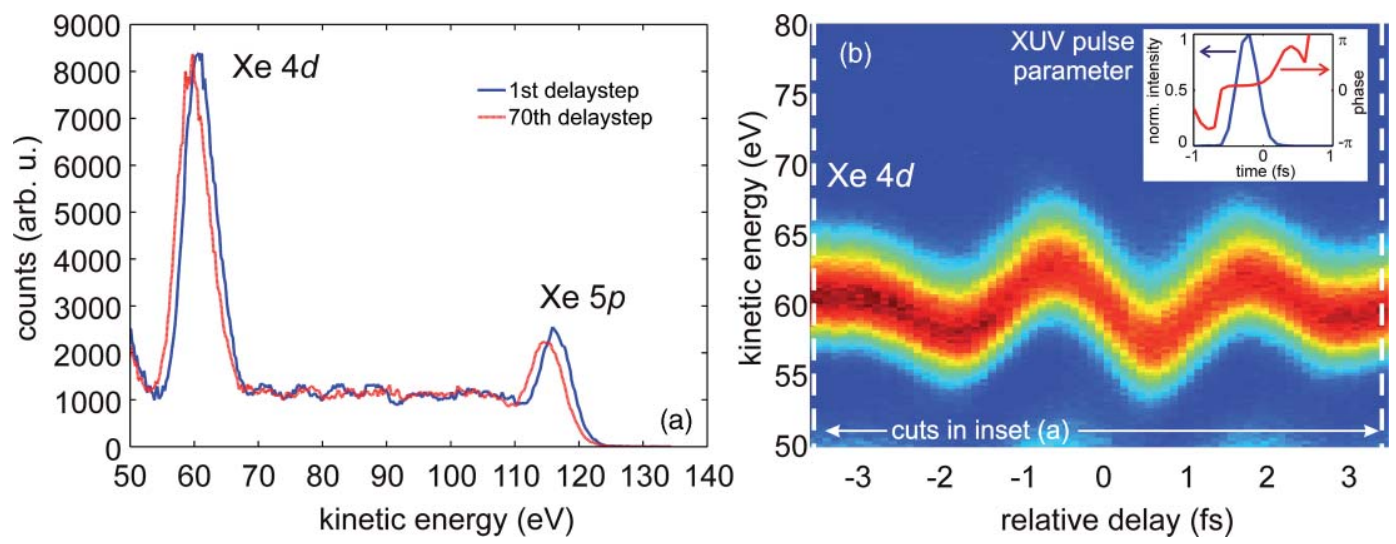

FIG. 4. (Color online) (a) Photoelectron spectra of a multilayer of xenon condensed on a $\operatorname{Re}(0001)$ substrate taken at the start and the end of a streaking scan with an integration time of $0.5 \mathrm{~min}$ ( $35 \mathrm{~min}$ total duration) illustrating the long-term stability of the xenon multilayer. (b) False-color plot showing the full streaking scan of the xenon $4 d$ state. The retrieved attosecond XUV pulse is shown in the small inset. The kinetic energy is referenced to the vacuum level, the spectra, and the spectrogram have been corrected for NIR-induced space charge effects.

condensate. A convolution of a high-resolution photoelectron spectrum obtained by synchrotron radiation with the photon energy dependent reflectivity of the multilayer mirror yields peak widths of $6.6 \mathrm{eV}$ and $6.7 \mathrm{eV}$ for the $5 p$ and $4 d$ peak, respectively (not shown). These values get slightly modified in the spectrogram in Fig. 4 due to the influence of the NIR streaking field and a non-zero chirp of the XUV pulse. ${ }^{13}$

All displayed photoelectron spectra were corrected for NIR-induced space charge effects. Space charge in the context here refers to energetically low photoelectrons, excited nonresonantly via multiple photo ionization by the NIR pulse, which accelerate the XUV photoelectrons towards the detector. In our case, this space charge leads to an overall shift of the kinetic energy of the XUV photoelectrons, which we correct for by adding an offset value derived from spectra obtained without the NIR field. This method is justified because the NIR focus on our sample is much larger than the XUV focus, i.e., the space charge effect is laterally homogeneous. A possible linear change of the amount of space charge during the acquisition of the spectrogram can be accounted for by fitting the effect with a linear function of $a+b$ t. As $b$ t turned out to be in the $\mathrm{meV}$ range, this term was not included in the correction.

The large bandwidth of the XUV pulse also sets limits to the minimal energy separation of two different electronic states, which are investigated with the ATR. In order to be able to extract information on their relative timing, ${ }^{8,41}$ both peaks have to be spectrally resolved, limiting the separation of the two states to at least the XUV pulse width. In reality, the XUV pulse will display a non-zero chirp which will cause a further broadening of the peaks when the NIR is introduced. ${ }^{13}$ Multiplets or a broad conduction band give rise to additional broadening. A general expression for this additional broadening cannot be given as it depends both on the XUV and NIR pulse parameters and on the investigated electronic structure of the sample.

Due to the UHV conditions, the signal strength of the photolines remained unchanged for more than $2 \mathrm{~h}$ - even at a substrate temperature of $20 \mathrm{~K}$. The xenon multilayers proved to be stable under simultaneous illumination of both the XUV and the NIR driver/streaking pulses enabling the ac- quisition of high quality streaking spectrograms ${ }^{13}$ as shown in Fig. 4(b). Due to the higher XUV photon energy, in comparison to previously reported streaking experiments, ${ }^{8}$ electronic states with a high binding energy like the $\mathrm{Xe} 4 d$ line are much less affected by the NIR-background photoelectrons, resulting from above-threshold ionization photoemission and inelastically scattered electrons.

The estimated NIR streaking intensity, based on the modulation depth of the photoelectron kinetic energies, ${ }^{12}$ is $\sim 4 \times 10^{11} \mathrm{~W} / \mathrm{cm}^{2}$. The high signal-to-noise ratio achieved in this measurement allows for accurate retrieval of important characteristics of the attosecond XUV pulses. The result of a frequency-resolved optical gating (FROG) routine applied to a xenon multilayer spectrogram is shown in the inset of Fig. 4(b). ${ }^{42}$ This analysis reveals an XUV pulse duration of 346 as with a central photon energy of $125 \mathrm{eV}$ and a group delay dispersion of $-9.2 \times 10^{3} \mathrm{as}^{2}$. The figure of merit of the algorithm is 0.23 , meaning the root-mean-square deviation between the retrieved and the measured spectrograms. ${ }^{42}$ To date, these are the highest energy XUV photons used in any attosecond streaking experiment.

\section{SUMMARY}

We describe a versatile apparatus dedicated to attosecond time-resolved photoelectron spectroscopy of solids and surfaces. The system is capable of generating sub-fs attosecond XUV pulses with energies ranging from 90 to $140 \mathrm{eV}$ and provides UHV conditions in a flexible end-station, where a wide range of standard surface science tools is available for preparation and characterization of well-defined surfaces and more sophisticated model systems. By performing an attosecond streaking experiment on a xenon multilayer, we demonstrate the long-term stability of our system, which allows us to record high quality streaking spectrograms even from a comparably fragile solid-state sample. Prolonged acquisition times, offered by the UHV environment, now enables streaking measurements to be performed on solid surfaces with equivalent quality to those made in simpler gas phase experiments. The flexibility of the end-station offers possibilities for both angle- and polarization-resolved attosecond electron 
spectroscopic experiments. Combined with the large variety of possible sample preparation schemes, this new apparatus constitutes the experimental basis for systematic studies of attosecond phenomena in solids and on surfaces. In the future, this apparatus can be further supplemented by a scheme to collinearly generate few-fs UV and attosecond XUV pulses ${ }^{43}$ allowing UV-XUV two-photon photoelectron spectroscopy in addition to attosecond streaking.

\section{ACKNOWLEDGMENTS}

The authors thank K. Kölbl, K. Eberle, and H. P. Schönauer for their valuable contributions to this work. Supported by the DFG Cluster of Excellence: Munich Centre for Advanced Photonics (www.munich-photonics.de). R.K. acknowledges funding from the Sofja Kovalevskaja Award of the Alexander von Humboldt Foundation and an ERC Starting Grant.

${ }^{1}$ C. Frischkorn and M. Wolf, Chem. Rev. 106(10), 4207 (2006).

${ }^{2}$ H. Petek and S. Ogawa, Prog. Surf. Sci. 56(4), 239 (1997).

${ }^{3}$ M. J. Weinelt, J. Phys.: Condens. Matter 14(43), R1099 (2002).

${ }^{4}$ A. Borisov, D. Sanchez-Portal, R. D. Muino, and P. M. Echenique, Chem. Phys. Lett. 387(1-3), 95 (2004).

${ }^{5}$ R. D. Muino, D. Sanchez-Portal, V. M. Silkin, E. V. Chulkov, and P. M. Echenique, Proc. Natl. Acad. Sci. 108, 971 (2011).

${ }^{6}$ C. Keller, M. Stichler, G. Comelli, F. Esch, S. Lizzit, W. Wurth, and D. Menzel, Phys. Rev. Lett. 80(8), 1774 (1998).

${ }^{7}$ A. Föhlisch, P. Feulner, F. Hennies, A. Fink, D. Menzel, D. Sanchez-Portal, P. M. Echenique, and W. Wurth, Nature (London) 436(7049), 373 (2005).

${ }^{8}$ A. L. Cavalieri, N. Müller, T. Uphues, V. S. Yakovlev, A. Baltuska, B. Horvath, B. Schmidt, L. Bluemel, R. Holzwarth, S. Hendel, M. Drescher, U. Kleineberg, P. M. Echenique, R. Kienberger, F. Krausz, and U. Heinzmann, Nature (London) 449(7165), 1029 (2007).

${ }^{9}$ S. Ogawa, H. Nagano, H. Petek, and A. P. Heberle, Phys. Rev. Lett. 78(7), 1339 (1997)

${ }^{10}$ A. L. Cavalieri, E. Goulielmakis, B. Horvath, W. Helml, M. Schultze, M. Fiess, V. Pervak, L. Veisz, V. S. Yakovlev, M. Uiberacker, A. Apolonski, F. Krausz, and R. Kienberger, New J. Phys. 9, 242 (2007).

${ }^{11}$ U. Graf, M. Fiess, M. Schultze, R. Kienberger, F. Krausz, and E. Goulielmakis, Opt. Express 16(23), 18956 (2008).

${ }^{12}$ J. Itatani, F. Quere, G. L. Yudin, M. Y. Ivanov, F. Krausz, and P. B. Corkum, Phys. Rev. Lett. 88(17), 173903 (2002).

${ }^{13}$ R. Kienberger, E. Goulielmakis, M. Uiberacker, A. Baltuska, V. Yakovlev, F. Bammer, A. Scrinzi, T. Westerwalbesloh, U. Kleineberg, U. Heinzmann, M. Drescher, and F. Krausz, Nature (London) 427(6977), 817 (2004).

${ }^{14}$ M. Uiberacker, T. Uphues, M. Schultze, A. J. Verhoef, V. Yakovlev, M. F. Kling, J. Rauschenberger, N. M. Kabachnik, H. Schröder, M. Lezius, K. L. Kompa, H. G. Muller, M. J. J. Vrakking, S. Hendel, U. Kleineberg, U. Heinzmann, M. Drescher, and F. Krausz, Nature (London) 446(7136), 627 (2007).

${ }^{15}$ M. Drescher, M. Hentschel, R. Kienberger, M. Uiberacker, V. S. Yakovlev, A. Scrinzi, T. Westerwalbesloh, U. Kleineberg, U. Heinzmann, and F. Krausz, Nature (London) 419(6909), 803 (2002).

${ }^{16}$ E. Goulielmakis, M. Uiberacker, R. Kienberger, A. Baltuska, V. S. Yakovlev, A. Scrinzi, T. Westerwalbesloh, U. Kleineberg, U.
Heinzmann, M. Drescher, and F. Krausz, Science 305(5688), 1267 (2004).

${ }^{17}$ A. Pietzsch, A. Fohlisch, M. Beye, M. Deppe, F. Hennies, M. Nagasono, E. Suljoti, W. Wurth, C. Gahl, K. Dobrich, and A. Melnikov, New J. Phys. 10, 033004 (2008).

${ }^{18}$ M. Stockman and M. F. Kling, Nat. Photonics 1, 539 (2007).

${ }^{19}$ A. Mikkelsen, J. Schwenke, T. Fordell, G. Luo, K. Klunder, E. Hilner, N. Anttu, A. A. Zakharov, E. Lundgren, J. Mauritsson, J. N. Andersen, H. Q. Xu, and A. L'Huillier, Rev. Sci. Instrum. 80, 123703 (2009).

${ }^{20}$ M. Fiess, M. Schultze, E. Goulielmakis, B. Dennhardt, J. Gagnon, M. Hofstetter, R. Kienberger, and F. Krausz, Rev. Sci. Instrum. 81, 093103 (2010).

${ }^{21}$ A. L'Huillier and Ph. Balcou, Phys. Rev. Lett. 70(6), 774 (1993).

${ }^{22}$ S. Hüfner, Photoelectron Spectroscopy (Springer-Verlag, Berlin, 1996).

${ }^{23}$ S. Mathias, L. Miaja-Avila, M. M. Murnane, H. C. Kapteyn, M. Aeschlimann, and M. Bauer, Rev. Sci. Instrum. 78(8), 083105 (2007).

${ }^{24}$ G. L. Dakovski, Y. Li, T. Durakiewicz, and G. Rodriguez, Rev. Sci. Instrum. 81, 073108 (2010).

${ }^{25}$ B. H. Christensen, M. K. Raarup, and P. Balling, Nucl. Instrum. Methods Phys. Res. A 615, 114 (2010).

${ }^{26}$ M. Drescher, M. Hentschel, R. Kienberger, G. Tempea, C. Spielmann, G. A. Reider, P. B. Corkum, and F. Krausz, Science 291, 1923 (2001).

${ }^{27}$ E. Constant, D. Garzella, P. Breger, E. Mevel, C. Dorrer, C. Le Blanc, F. Salin, and P. Agostini, Phys. Rev. Lett. 82(8), 1668 (1999).

${ }^{28} \mathrm{G}$. Tempea, M. Geissler, M. Schnürer, and T. Brabec, Phys. Rev. Lett. 84(19), 4329 (2000)

${ }^{29}$ A. Baltuska, T. Udem, M. Uiberacker, M. Hentschel, E. Goulielmakis, C. Gohle, R. Holzwarth, V. S. Yakoviev, A. Scrinzi, T. W. Hänsch, and F. Krausz, Nature (London) 421(6923), 611 (2003).

${ }^{30}$ P. B. Corkum, Phys. Rev. Lett. 71(13), 1994 (1993).

${ }^{31}$ M. Lewenstein, P. Balcou, M. Y. Ivanov, A. Lhuillier, and P. B. Corkum, Phys. Rev. A 49(3), 2117 (1994).

${ }^{32}$ N. Nakano, H. Kuroda, T. Kita, and T. Harada, Appl. Opt. 23(14), 2386 (1984).

${ }^{33}$ T. Kita, T. Harada, N. Nakano, and H. Kuroda, Appl. Opt. 22(4), 512 (1983).

${ }^{34}$ T. A. Johnson, R. Soufli, E. M. Gullikson, and M. Clift, Proc. SPIE 5538, 119 (2004).

${ }^{35}$ E. Palik, Handbook of Optical Constants (Academic, New York, 1991).

${ }^{36}$ B. L. Henke, E. M. Gullikson, and J. C. Davis, At. Data Nucl. Data Tables 54(2), 181 (1993).

${ }^{37}$ A. Wonisch, U. Neuhausler, N. M. Kabachnik, T. Uphues, M. Uiberacker, V. S. Yakovlev, F. Krausz, M. Drescher, U. Kleineberg, and U. Heinzmann, Appl. Opt. 45(17), 4147 (2006).

${ }^{38}$ M. Hofstetter, M. Schultze, M. Fiess, A. Guggenmos, J. Gagnon, E. Magerl, E. Bothschafter, R. Ernstorfer, R. Kienberger, E. M. Gullikson, F. Krausz, and U. Kleineberg, in Prooceedings of Postdeadline Paper of the Ultrafast Phenomena XVII, paper PDP8, 2010.

${ }^{39}$ R. Opila and R. Gomer, Surf. Sci. 112, 1 (1981).

${ }^{40}$ M. Stindtmann, M. Farle, T. S. Rahman, L. Benabid, and K. Baberschke, Surf. Sci. 381(1), 12 (1997).

${ }^{41}$ M. Schultze, M. Fiess, N. Karpowicz, J. Gagnon, M. Korbman, M. Hofstetter, S. Neppl, A. L. Cavalieri, Y. Komninos, T. Mercouris, C. A. Nicolaides, R. Pazourek, S. Nagele, J. Feist, J. Burgdoerfer, A. M. Azzeer, R. Ernstorfer, R. Kienberger, U. Kleineberg, E. Goulielmakis, F. Krausz, and V. S. Yakovlev, Science 328(5986), 1658 (2010).

${ }^{42}$ J. Gagnon, E. Goulielmakis, and V. S. Yakovlev, Appl. Phys. B 92(1), 25 (2008).

${ }^{43}$ E. M. Bothschafter, A. Schiffrin, V. S. Yakovlev, A. M. Azzeer, F. Krausz, R. Ernstorfer, and R. Kienberger, Opt. Express 18, 9173 (2010). 